\title{
Comparison of Step-and-Shoot and Continuous-Bed-Motion PET Modes of Acquisition for Limited-View Organ Scans
}

\author{
Wendy $\operatorname{Siman}^{1,2}$ and S. Cheenu Kappadath ${ }^{3,4}$ \\ ${ }^{1}$ Department of Radiology, University of Tennessee Medical Center, Knoxville, Tennessee; ${ }^{2}$ University of Tennessee Graduate School \\ of Medicine, Knoxville, Tennessee; ${ }^{3}$ Department of Imaging Physics, University of Texas MD Anderson Cancer Center, Houston, \\ Texas; and ${ }^{4}$ University of Texas Graduate School of Biomedical Sciences, Houston, Texas
}

\begin{abstract}
Continuous-bed-motion (CBM) acquisition mode has been made commercially available in PET/CT scanners. CBM mode is designed for whole-body imaging, with a long scan length (multiple axial fields of view [aFOVs]) and short acquisition duration (2-3 min/aFOV). PET/CT has recently been used after ${ }^{90}$ Y-microsphere therapy to quantify ${ }^{90} \mathrm{Y}$ activity distribution in the liver. Here we compared counting efficiencies along the bed-motion direction (z-axis) between CBM and step-and-shoot (SS) acquisition modes for limited-view organ scans, such as ${ }^{90} \mathrm{Y}$ PET/CT liver studies, that have short scan lengths ( $\leq 2 \mathrm{aFOVs}$ ) and long acquisition durations (10-30 min/aFOV). Methods: The counting efficiencies, that is, analytic sensitivities, in SS mode (single-aFOV and multiple-aFOV scans) and CBM mode were theoretically derived and experimentally validated using a cylindric ${ }^{68} \mathrm{Ge}$ phantom. The sensitivities along the $z$-axis were compared between the SS and CBM modes. Results: The analytic and experimental count profiles were in good agreement, validating the analytic models. For fixed scan durations, the overall coincidence counting efficiency in CBM mode was lower ( $60 \%)$ than those in SS modes, and the maximum sensitivity in CBM mode was $50 \%$ or less of that in 1 -aFOV SS mode and $100 \%$ or less of that in 2 -aFOV SS mode. Conclusion: The ability of CBM mode to tailor-fit the PET/CT scan length and local scan duration is not realized in studies with a short scan length $(\leq 30 \mathrm{~cm})$ and long scan duration $(20 \mathrm{~min} / \mathrm{aFOV}$ for the scanner). SS acquisition mode is preferable to CBM mode for limitedview organ and count-starved scans, such as ${ }^{90 Y}$ PET/CT liver scans, because of the higher counting efficiency of SS mode, which leads to better image quality and quantification precision.
\end{abstract}

Key Words: continuous bed motion; step and shoot; ${ }^{90}$ Y-microsphere; PET/CT

J Nucl Med Technol 2017; 45:290-296

DOI: 10.2967/jnmt.117.195438

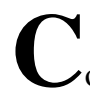

ontinuous-bed-motion (CBM) mode was originally developed to improve axial image uniformity in wholebody imaging in 2-dimensional step-and-shoot (SS) acquisition mode (1), which has sensitivity variations in the axial

\footnotetext{
Received Apr. 29, 2017; revision accepted Aug. 31, 2017.

For correspondence or reprints contact: S. Cheenu Kappadath, Department of Imaging Physics, University of Texas MD Anderson Cancer Center, 1515 Holcombe Blvd., Houston, TX 77030.

E-mail: skappadath@mdanderson.org

Published online Oct. 17, 2017.

COPYRIGHT (c) 2017 by the Society of Nuclear Medicine and Molecular Imaging.
}

direction due to gaps between detector blocks (2). However, axial uniformity is no longer a problem in modern PET/CT scanners using a 3-dimensional (3D) SS acquisition mode, in which axial uniformity can be improved by overlapping the end slices of adjacent bed positions and combining the overlapped data (3). Theoretically, CBM mode may also allow oversampling in the $z$-axis direction and produce superresolution images (4); however, this superresolution effect is not realized in clinical practice because of a high level of noise (2).

The true clinical benefit of CBM is the ability to tailor-fit the PET/CT scan length and local scan duration to the imaging task. In traditional SS mode, the PET scan length increases in steps of the PET $z$-axis field of view (aFOV) depending on the amount of detector overlap between bed positions. Hence, the ability to tailor-fit the PET/CT scan length allows modest CT dose saving and facilitates trading off the scan length with the total scan duration. In multibed SS acquisitions, the PET acquisition duration per bed position is usually fixed; whereas in CBM mode, the local acquisition duration can be modulated along the $z$-axis and adjusted by varying the bed speed, allowing acquisition of higher counts in regions of greater interest. In addition, clinical practice has suggested that patients find CBM mode more comfortable than SS acquisition $(5,6)$.

Recently, CBM acquisition mode has been made commercially available in the Siemens Biograph mCT Flow (Siemens Medical Solutions USA). CBM mode is intended for wholebody PET/CT imaging with a long scan length (multiple aFOVs). Multiple studies have suggested that the image quality in clinical whole-body imaging using CBM is at least comparable to SS mode, with CBM mode having better endplane image quality $(2,6,7)$. However, the performance of CBM mode for shorter scan lengths, such as liver or brain PET scans for which the scan length is typically less than 20 $30 \mathrm{~cm}$, has not been investigated. The objective of the present work was to compare the counting efficiency per axial slice, that is, the slice sensitivity profile realized along the $z$-axis between CBM and SS modes of acquisition for equal scan duration in limited-view organ scans. We also evaluated the implications of these differences for the image quality and quantification of a ${ }^{90} \mathrm{Y}$ PET/CT liver scan, for which the scan length is typically less than $30 \mathrm{~cm}$ and the scan duration is typically $20-30 \mathrm{~min} /$ bed position $(8,9)$. 


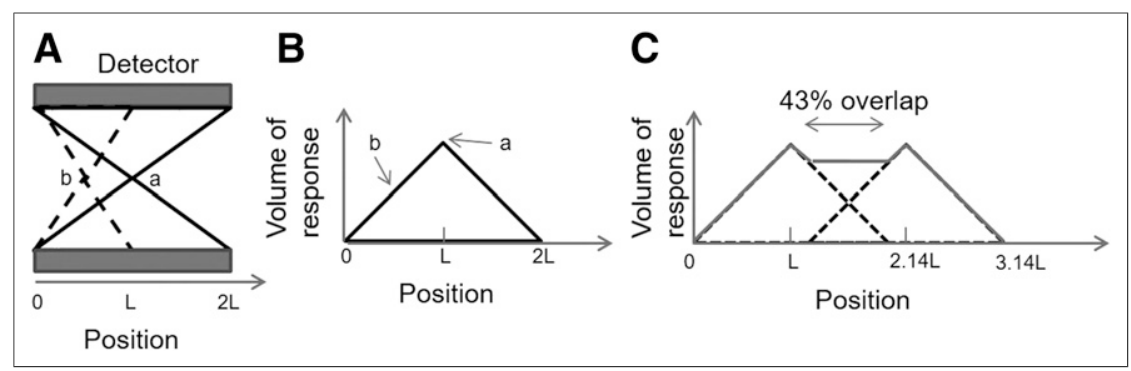

FIGURE 1. An idealized axial slice sensitivity profile to conceptually illustrate slice sensitivity/bed overlap in 3D SS modes. (A) VOR in 3D SS acquisition at 2 locations: $a$ and $b$. VOR varies linearly as function of distance along $z$-axis aFOV. (B) For 1 aFOV, VOR and slice sensitivity as function of position have triangular profile. (C) For 2-aFOV SS acquisition, VOR profile as function of position is simply summation of individual aFOV profiles at each bed position taking into account amount of bed overlap; $43 \%$ aFOV overlap in Siemens Biograph mCT Flow is illustrated.

\section{MATERIALS AND METHODS}

\section{Analytic Comparison of Counting Efficiency Between CBM and SS Modes}

The slice sensitivity profile of PET acquisition is proportional to the volume of response (VOR) accepted for a given geometry (10). The number of counts in an image slice is proportional to the integral of its VOR over time. For a uniformly distributed activity along the bed-motion direction (z-axis), the $z$-axis count profile of the image slices $z, \mathrm{C}(z)$, can be calculated as

$$
\mathrm{C}(z) \propto \int_{0}^{\mathrm{t}_{\mathrm{dur}}} \operatorname{VOR}(z, t) \mathrm{d} t
$$

where $\operatorname{VOR}(z, t)$ is the $\operatorname{VOR}$ at the location of image slice $z$ and time $t$, and $t_{d u r}$ is the total acquisition duration. Differences in the slice sensitivity profile between SS and CBM are dominated by differences in their scan geometry, and to a smaller degree by subtle variations in the efficiency of coincidence event detection along the axial direction due to postprocessing aspects of coincidence detection and variations in scintillator efficiency and system electronics $(10,11)$. Derivation of the slice sensitivity profile in this work is solely based on the geometric factors stemming from differences in SS and CBM scan modes.

Slice Sensitivity in 3D SS Mode. In 3D SS acquisition mode, the VOR at any given axial position along the PET bed is proportional to its distance from the edge of the aFOV - that is, the VOR is largest at the center (L) and smallest at the edges ( 0 or $2 \mathrm{~L}$ ) of the aFOV (Fig. 1A). Three-dimensional SS acquisition therefore has a triangular slice sensitivity profile for a scan length of $1 \mathrm{aFOV}$ (2L), as shown in Figure 1B. In reality, the slice sensitivity profile is not exactly triangular but has a sawtooth shape with a plateau depending on the degree of axial compression (reducing the number of sinograms that have a central line of response intersecting the scanner axis at the same location), the maximum acceptance angle, and the number of detectors used on the scanner $(2,10)$. For multi-aFOV SS acquisition, the aggregate axial slice sensitivity profiles can be calculated by adding the count profiles of each individual aFOV acquisition while accounting for the amount of bed overlap (Fig. 1C).

In 1-bed SS mode, the VOR varies only as a function of location inside the aFOV; hence, the count profile from Equation 1 simply becomes $\mathrm{C}(z) \propto \operatorname{VOR}(z) \times t_{\text {dur }}$. In 2-bed SS (or multibed SS), the VOR varies with time per bed position as the scanner steps and shoots; hence, the 2-bed SS count profile calculated using Equation 1 is $\mathrm{C}(z) \propto\left\{\left.\operatorname{VOR}(z)\right|_{\text {bed } 1} \times\right.$ $\left.t_{\text {bed } 1}+\left.\operatorname{VOR}(z)\right|_{\text {bed } 2} \times t_{\text {bed } 2}\right\}$ (or equivalently, $\mathrm{C}(z) \propto\left\{\left[\left.\operatorname{VOR}(z)\right|_{\text {bed } 1}+\left.\operatorname{VOR}(z)\right|_{\text {bed } 2}\right] \times t_{\text {bed }}\right\}$ when $t_{\text {bed }}$ is the same for both bed locations), where the first and second term are the left and right triangles, respectively, in Figure 1C. The total sensitivity for a specific $z$ location is cumulative if it is present in multiple SS scans.

Slice Sensitivity Profile in CBM Mode. In contrast to the SS mode variation of $\operatorname{VOR}(z, t)$, which is discrete in its axial profile for each bed, $\operatorname{VOR}(z, t)$ in $\mathrm{CBM}$ mode depends on how the slice moves across the detector.

Figure 2 shows the variation of $\operatorname{VOR}_{\mathrm{CBM}, z}(t)$ at slice $z$ (initial location at $L / 2$ from the edge of the aFOV) as the bed translates into the gantry. The slice sensitivity at slice $z$ is proportional to the acquired counts in slice $z, \mathrm{C}(z)$, which can be calculated as the integration over time of the $\operatorname{VOR}_{\mathrm{CBM}, z}(t)$ according to Equation 1. Alternatively, $\mathrm{C}(z)$ can be calculated in a straightforward manner as the area under the curve of $\operatorname{VOR}_{\mathrm{CBM}, z}(t)$ as shown in Figure 2 (shaded area). Locations that start outside the aFOV will travel the entire length of the aFOV and have the highest slice sensitivity, that is, the area under the entire triangular profile as realized in SS mode. In contrast, locations that start inside the aFOV will, depending on their relative positions, travel a shorter length inside the aFOV and have a lower slice sensitivity.

For the Siemens Biograph mCT Flow scanner, CBM mode is acquired with $50 \%$ overscan, in which the center of the detector aFOV begins and ends at extremes of the scan prescription (2). In this configuration, the count profile for a uniform cylindric source consists of ramping-up and -down regions, each half an aFOV long at the start and end with a flat profile in between the ramping regions (Fig. 3). The minimum counting efficiency occurs at bed locations that travel half of the aFOV, that is, between locations near the start and end of the scan prescription, with maximum efficiency at locations that are at the edge of the aFOV during the start and end of CBM. Mathematically, the slice sensitivity profiles, which are proportional to the slice sensitivity profiles, can be expressed in CBM mode as:

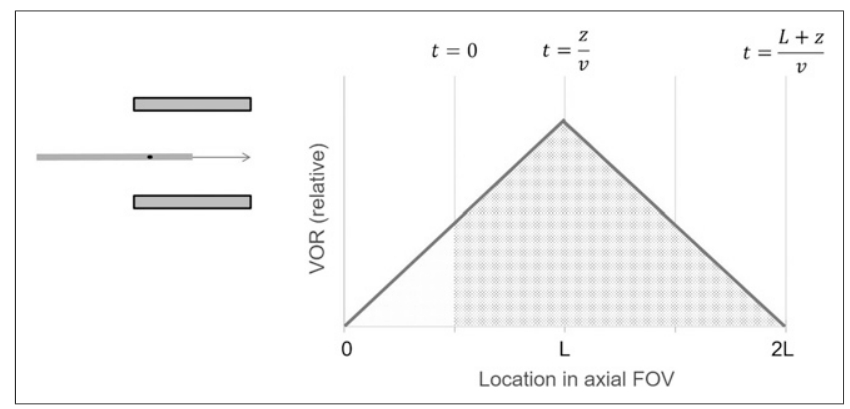

FIGURE 2. Variation of VOR at slice $z$ (black dot) of line source (thick gray line) as it translates into scanner aFOV. Pathlength of this slice across aFOV is shown by arrow in left panel. Slice sensitivity of slice $z$ here can be calculated as area under curve (shaded region) of VOR. 


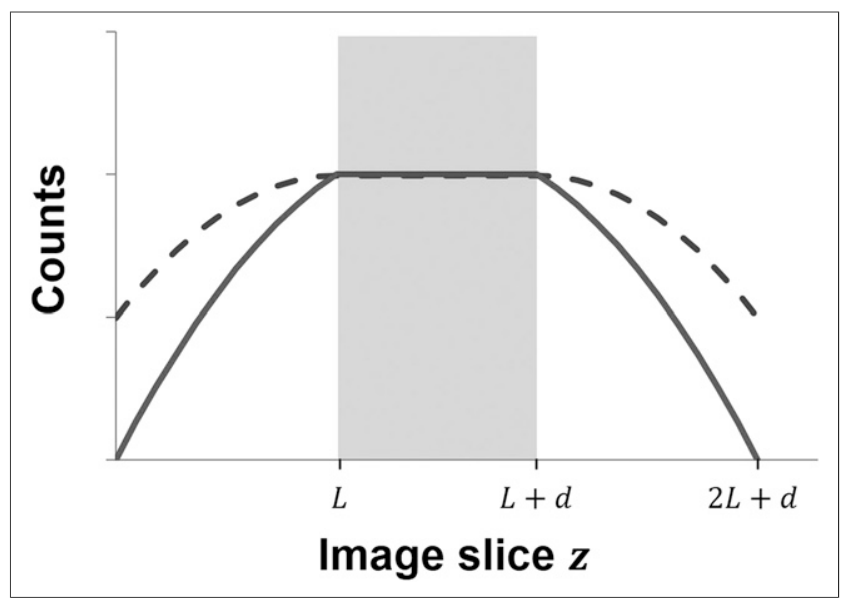

FIGURE 3. Count profile at location $z$, that is, $\mathrm{C}(z)$ in CBM (dashed line) and $\mathrm{CT}$-limited $\mathrm{CBM}^{\prime}$ mode (solid line). Counts are lower in $\mathrm{CBM}^{\prime}$ at ramping-up and -down regions, where CT attenuation-correction map is incomplete. CT attenuationcorrection map is complete only in flat region between $L$ and $\mathrm{L}+\boldsymbol{d}$ (gray region). Profiles are derived for CBM acquisition with $50 \%$ overscan.

$$
\begin{gathered}
\mathrm{S}(z) \propto-\frac{z^{2}}{2 \mathrm{~L} v}+\frac{z}{v}+\frac{\mathrm{L}}{2 v} \quad \text { for } 0 \leq z \leq \mathrm{L}, \\
\mathrm{S}(z) \propto \frac{\mathrm{L}}{v} \quad \text { for } \mathrm{L} \leq z \leq \mathrm{L}+d, \\
\mathrm{~S}(z) \propto-\frac{z^{\prime 2}}{2 \mathrm{~L} v}+\frac{z^{\prime}}{v}+\frac{\mathrm{L}}{2 v} \quad \text { for } \mathrm{L}+d \leq z \leq 2 \mathrm{~L}+d,
\end{gathered}
$$

Eq. 2

where $d$ is the length of the uniform region, $v$ is the bed speed, and $z^{\prime}=-(z-2 \mathrm{~L}-d)$ (Fig. 3). Note that $d$ is equal to the total scan length prescription minus $2 \mathrm{~L}$. In reality, the sensitivity profile depends on a large number of hardware and software parameters that include the detector segments used and the scan extent outside the aFOV. We have once again simplified the theoretic derivation to conceptually illustrate the slice sensitivity profile in CBM mode. Figure 3 graphs the count profiles of $\mathrm{CBM}$ and $\mathrm{CBM}^{\prime}$ modes.

Like SS mode, the CBM acquisition in the Siemens Biograph mCT Flow has matching scan length prescriptions for PET and CT. The implementation of CBM mode on this scanner incorporates $50 \%$ aFOV overscan outside the PET acquisition prescription; hence, a CT attenuation-correction map is missing in the overscan regions. Consequently, the coincidence counts in VOR that include these outside locations cannot be corrected for attenuation and used in the quantitative PET image reconstruction (2). In CT-limited CBM mode (henceforth referred to as $\left.\mathrm{CBM}^{\prime}\right), \operatorname{VOR}(t)$ has a modified trapezoidal shape (Fig. 4). The plateau occurs because the useful VOR is limited by the missing CT attenuation-correction map even though the geometric VOR grows. Note that in SS acquisition mode, all PET data have a complete $\mathrm{CT}$ attenuation-correction map because of the matching PET/CT acquisition prescription.

As illustrated in Figure 4, the PET VOR and therefore the count profile at the axial edges of the prescribed scan regions are limited by the unavailability of CT attenuation correction rather than by the acquisition geometry. Like in CBM mode, following Equation 1, the count profile for $\mathrm{CBM}^{\prime}$ mode can be expressed as:

$$
\begin{gathered}
\mathrm{S}(z) \propto \frac{3 z}{2 v}-\frac{z^{2}}{2 \mathrm{~L} v}, \quad \text { for } 0 \leq z \leq \mathrm{L}, \\
\mathrm{S}(z) \propto \frac{\mathrm{L}}{v}, \quad \text { for } \mathrm{L} \leq z \leq \mathrm{L}+\mathrm{d}, \\
\mathrm{S}(z) \propto \frac{3 z^{\prime}}{2 v}-\frac{z^{\prime 2}}{2 \mathrm{~L} v}, \quad \text { for } \mathrm{L}+d \leq z \leq 2 \mathrm{~L}+\mathrm{d}, \quad \text { Eq. } 3
\end{gathered}
$$

Note that contrary to CBM mode, in $\mathrm{CBM}^{\prime} \mathrm{S}(0)=0$.

Analytic Comparison of Slice Sensitivity Profile Between SS and CBM Modes. We compared the counting efficiencies of PET data acquisition in 1-aFOV and 2-aFOV 3D SS modes and CBM mode for the same duration. For the 2-aFOV scan, the acquisition duration for each bed position was half the acquisition duration in the $1-\mathrm{aFOV}$ scan to maintain the same total duration. The scan lengths in $1-\mathrm{aFOV}$, 2-aFOV, and CBM modes were 22,35 , and $22 \mathrm{~cm}$, respectively.

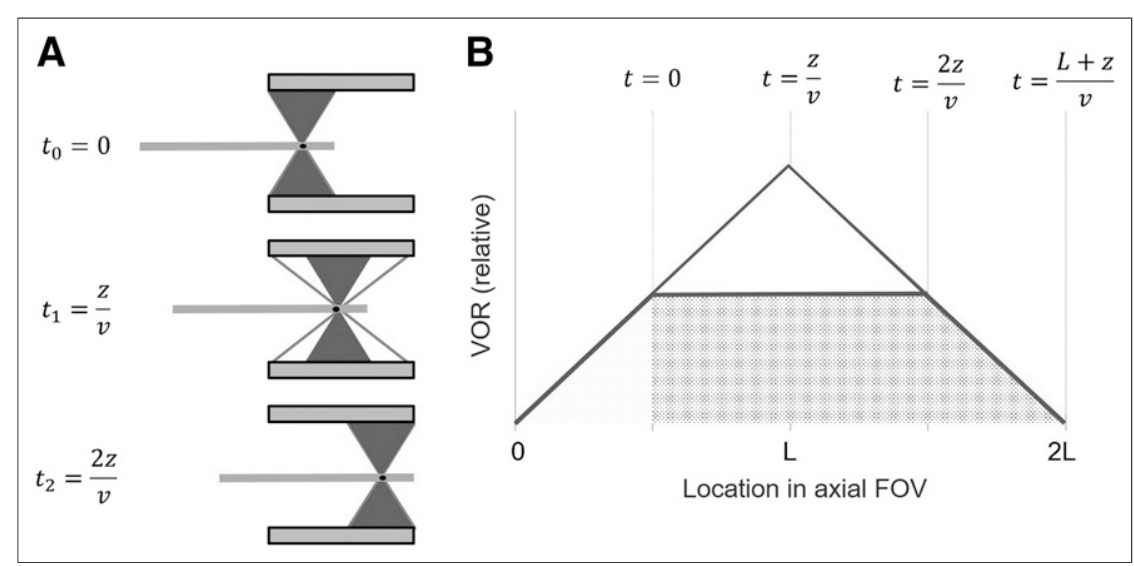

FIGURE 4. (A) Comparison of variation of VOR at slice $z$ (black dot) of line source (thick gray line) between CBM (empty triangles) and CT-limited CBM or CBM' (gray triangles). (B) Graph of VOR at slice $z$ as function of time $t$ (or location in aFOV) in CBM and $\mathrm{CBM}^{\prime}$ (shaded region).

\section{Experimental Verification of Slice Sensitivity Profiles in SS and CBM Modes}

All PET/CT images were acquired using the Biograph mCT Flow scanner featuring the CBM acquisition mode (Flow Motion). The scanner has a bore diameter of $78 \mathrm{~cm}$ and an aFOV of $22 \mathrm{~cm}$ (4-ring detector configuration). The system is also time-of-flight capable and has a nominal timing resolution of $0.555 \mathrm{~ns}$.

A 74-MBq (2-mCi) (nominal) ${ }^{68} \mathrm{Ge}$ daily quality control phantom with a length of $270 \mathrm{~mm}$ and a diameter of $200 \mathrm{~mm}$ was scanned using the 1-aFOV SS, 2-aFOV $\mathrm{SS}$, and CBM PET acquisition modes to derive the count density profiles of each mode. The scan prescriptions for all acquisition modes are shown in Supplemental Figure 1 
(supplemental materials are available at http://tech.snmjournals. org). The acquisition parameters were the same as those used for analytic comparison.

All PET/CT images were reconstructed using time-of-flight information with CT attenuation correction, point-spread-function modeling, scatter correction, and ordered-subset expectation maximization iterative reconstruction (TrueX; Siemens Medical Solutions USA) with 21 subsets and 2 iterations.

The slice sensitivity in the axial direction (bed-motion direction) for each acquisition mode was computed as the total slice counts as a function of slice position. The full-length count profiles $(220 \mathrm{~mm})$ were calculated for the $1-\mathrm{aFOV}$ SS and CBM modes, whereas for the 2 -aFOV SS mode, the count profiles were calculated for half the FOV $(173 \mathrm{~mm})$ because the ${ }^{68} \mathrm{Ge}$ phantom was shorter $(270 \mathrm{~mm})$ than the full 2 aFOVs $(345.5$ $\mathrm{mm})$. These relative slice sensitivity profiles were then compared with the profiles derived analytically as described above for validation.

\section{RESULTS}

The theoretically derived and experimentally measured count density profiles are shown for 1 -aFOV and 2 -aFOV SS modes and CBM mode in Figure 5A. The measured profiles for 1-aFOV mode and CBM mode with axial scan lengths of $22 \mathrm{~cm}$ were in good agreement with the theoretically derived profiles. The measured profile for 2-aFOV mode with an axial scan length of $35.4 \mathrm{~cm}$ was characterized for half the axial extent because the phantom used for the measurements was shorter in length (only $\sim 27 \mathrm{~cm}$ ).

In Figure 5B, the computed count profile for the 2-aFOV SS mode is mirror-imaged (dashed lines) to give a full 2aFOV profile. The slice sensitivity profile in CBM mode in the ramping-up and -down region resembles the steps function because of sinogram chucking (2) (data segmentation that allows image reconstruction during data acquisition). As a result of sinogram chucking, we also observed a flat
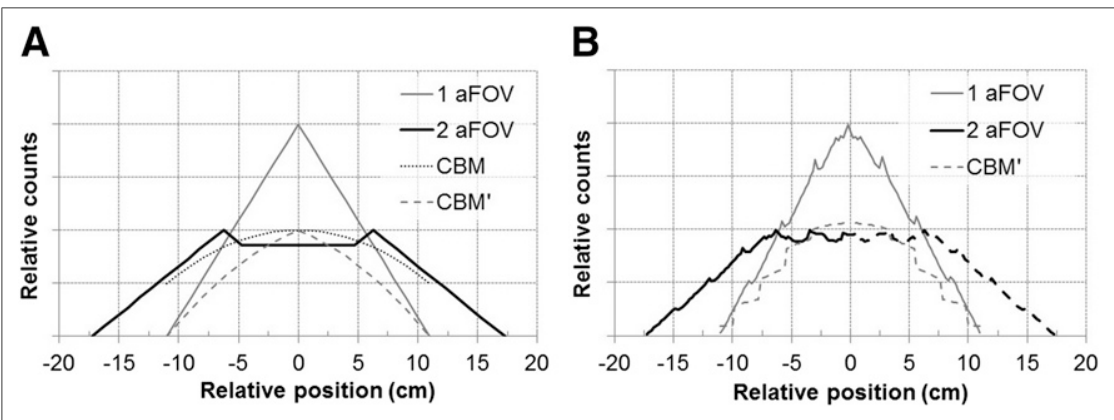

FIGURE 5. (A) Comparison of analytically derived counting efficiencies for uniform line cylindric source acquired using SS mode (scan lengths of 1 and 2 aFOVs) and CBM mode. All profiles are aligned at central slices to compare tradeoffs between counts and scan length. CBM profile (gray dotted line) shows slice sensitivity for geometry-limited CBM mode, whereas $\mathrm{CBM}^{\prime}$ profile (gray dashed line) shows slice sensitivity realized in CBM mode, with missing attenuation correction in overscan region. (B) Experimentally derived count profiles for 1aFOV and 2-aFOV SS modes and CBM mode. Black dashed line for 2-aFOV curve indicates mirrored profile. region in the 1-aFOV $\mathrm{CBM}^{\prime}$ profile (Fig. 5B) even though geometry-based derivation (Fig. 5A) indicates that the 1 -aFOV CBM profile would not have a flat region. Nonetheless, agreement between measured and theoretic profiles for 2-aFOV SS scans can be fully appreciated in the positions in which the phantom extends fully (solid line).

In the CBM implementation in the Biograph mCT Flow, because of partially unusable data (missing attenuationcorrection factor) in the overscan region, the observed slice sensitivity was even lower than in the SS modes. For the same scan durations, the overall slice sensitivity in CBM mode was lower than those of $1-\mathrm{aFOV}$ and 2 -aFOV SS modes in terms of total PET signal (area under the curves). CBM mode acquired only approximately $80 \%$ (CBM) and $60 \%\left(\mathrm{CBM}^{\prime}\right)$ of the total counts acquired in the SS modes for the same acquisition duration. In addition, for the same acquisition duration, the ratio of the maximum slice sensitivity in CBM mode to that in 1-aFOV SS mode was proportional to $\mathrm{aFOV} /\left(2 \times\right.$ scan length $\left._{\mathrm{CBM}}\right)$-that is, the slice counts in the CBM were $50 \%$ or less of those in 1 -aFOV SS mode and $100 \%$ or less of those in 2 -aFOV SS mode.

\section{DISCUSSION}

In this study, for fixed scan durations, the overall coincidence counting efficiency and maximum slice sensitivity in CBM mode were substantially lower than those in SS modes, indicating that SS mode is preferable to CBM (and $\mathrm{CBM}^{\prime}$ ) mode for limited-view organ and count-starved scans.

\section{Effects of Acquisition Modes on Image Quality and Quantitation}

In our previous work (12), we have shown that the errors in $3 \mathrm{D}$ dose-activity concentration quantification are directly affected by the total number of counts acquired in PET. Visually we have also shown that image noise, which is governed by the number of counts, alters the 3D dose-activity distribution. Therefore, to optimize both image quality and dose-activity quantification, we need to maximize the number of counts collected during PET acquisition.

Both SS and CBM PET acquisition modes allow for trade-off between the counts per unit, (axial) distance, and scan length for fixed acquisition duration. With SS mode, the increments in scan length are in a fixed fraction of PET aFOV depending on the prescribed bed overlap; with CBM, however, the scan length can be increased in increments of the allowed table speed. In our study, we found that for the same acquisition duration, the maximum sensitivity (at the central 
images) for CBM mode is less than or equal to half of that for the 1-aFOV SS mode and less than or equal to the maximum slice sensitivity for the 2 -aFOV SS mode. For the same scan length and scan duration, CBM mode collected only $60 \%$ of the coincidence counts that were collected in the 1 -aFOV SS mode. For the same acquisition duration, the maximum slice sensitivity of a 2-aFOV SS acquisition relative to 1 -aFOV SS mode depends on the amount of bed overlap; for $50 \%$ bed overlap, the maximum slice sensitivity in 2-aFOV SS mode is half of that in 1-aFOV mode.

Because SS mode has a higher slice sensitivity than CBM mode for a short-length scan, we recommend using SS mode instead of CBM mode for studies such as a liver scan after ${ }^{90} \mathrm{Y}$-microsphere therapy.

\section{Parameters Affecting CBM Mode}

The CBM and $\mathrm{CBM}^{\prime}$ sensitivity profiles, as shown in Equations 2 and 3, are inversely proportional to bed speed $(v)$, which is an adjustable acquisition parameter. For a given scan length, the effect of changing the bed speed in CBM mode is analog to changing the time/bed in SS mode; both parameters change only the magnitude of the profile. Supplemental Figure 2 shows CBM profiles for 1 -aFOV $(22 \mathrm{~cm})$ with bed speeds of $0.1,0.3$, and 0.5 $\mathrm{mm} / \mathrm{s}$. For a Biograph mCT Flow, $0.1 \mathrm{~mm} / \mathrm{s}$ is the slowest bed speed and the smallest bed-speed increment available in CBM mode.

The shape of $\mathrm{CBM}$ and $\mathrm{CBM}^{\prime}$ profiles in the ramp-up and ramp-down regions depends on the amount of overscan. At $50 \%$ overscan, the $\mathrm{CBM}$ and $\mathrm{CBM}^{\prime}$ profiles consist of $1 / 2 \mathrm{aFOV}$ ramp-up and $1 / 2 \mathrm{aFOV}$ ramp-down regions. In this configuration, the bed-motion extent is equal to the total scan length. As the amount of overscan increases beyond $50 \%$, the bed has to travel longer than the scan length prescription. Consequently, for a fixed scan duration the bed speed has to be increased, resulting in lower slice sensitivity profiles (Fig. 6). At $100 \%$ overscan, the CBM profile consists of a uniform profile with no ramp-up/-down regions, albeit the sensitivity profile is lower than the profiles with less amount of overscan.

In the $\mathrm{CBM}^{\prime}$ case, the useful data in the overscan regions are limited by the $\mathrm{CT}$ attenuation-correction availability;

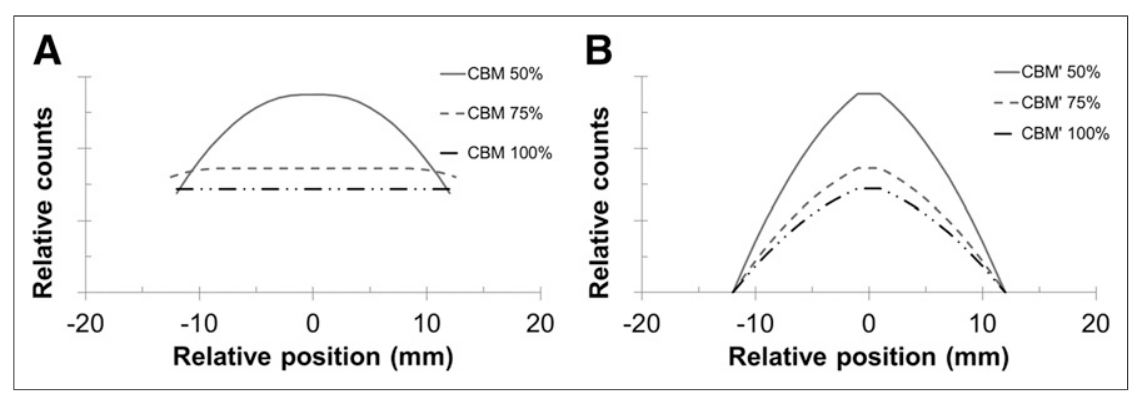

FIGURE 6. $\mathrm{CBM}(\mathrm{A})$ and $\mathrm{CBM}^{\prime}(\mathrm{B})$ profiles as function of degree of overscan. as a result, increasing the overscan range only results in decrease of the slice sensitivity profiles.

\section{CBM Versus SS Modes: Tailor-Fitting ${ }^{90}$ Y PET Scan Protocol}

The primary advantages of CBM mode over SS mode are that CBM mode offers a uniform axial slice sensitivity profile and facilitates effective trading between scan length and scan duration. In this study, we found that these features were not realized in scans of limited axial coverage and long scan duration, such as after ${ }^{90} \mathrm{Y}$-microsphere therapy liver imaging.

As shown in Figures 4 and 5, the CBM slice sensitivity profile was not uniform for the first and the last halves of the aFOV. For a scan length of 2 or fewer aFOVs, these nonuniform regions comprised $50 \%$ or more of the total scan length.

In practice, the adjustment of the scan duration in CBM mode is restricted, especially in a long-scan-duration study, by the finite increments of table speed. For example, the typical scan protocol for a post- ${ }^{90}$ Y-microsphere PET study has an axial coverage of $24 \mathrm{~cm}$ (based on patient population at our institution), with total scan duration of up to $40 \mathrm{~min}$ (2 bed positions). A scan with an axial extent of $24 \mathrm{~cm}$ can also be covered in 40 min using CBM mode with the slowest bed speed of $0.1 \mathrm{~mm} / \mathrm{s}$ in a Biograph mCT Flow. However, because the next available speed setting is $0.2 \mathrm{~mm} / \mathrm{s}$, the next available scan duration for a $24-\mathrm{cm}$ coverage is $20 \mathrm{~min}$. On the other hand, the finest scan-duration increment of $1 \mathrm{~s}$ in SS mode makes it more flexible in tailor-fitting the scan duration for long-duration PET acquisitions.

Because of its finite bed speed increments, in a count-limited PET/CT study (e.g., ${ }^{90} \mathrm{Y}$ imaging), the CBM mode loses its ability to adjust local scan duration, that is, flexible trade-off between scan lengths and duration.

Scan length adjustment in SS mode is limited by the bed steps, which is in the increments of $12.54 \mathrm{~cm}$ for scanners used in this study (22-cm aFOV, $43 \%$ bed overlap). If the anatomic region of interest is $26 \mathrm{~cm}$, the patient has to be scanned using 2-aFOV SS mode with an axial coverage of $34.5 \mathrm{~cm}$. The scan length can be prescribed to exactly 26 $\mathrm{cm}$ with the same scan duration using CBM mode. However, tailor-fitting the scan length using CBM mode is not necessary for scan lengths of less than $30 \mathrm{~cm}$ because SS mode provides higher overall slice sensitivity and longer coverage at the same time. Figure 7A demonstrates that 1.5-aFOV CBM mode (26-cm axial extent) offers a counting sensitivity (both magnitude and coverage) inferior to 2 -aFOV SS mode (34.5-cm axial extent). The slice sensitivity profile in 2 -aFOV SS mode is at least comparable in magnitude to 


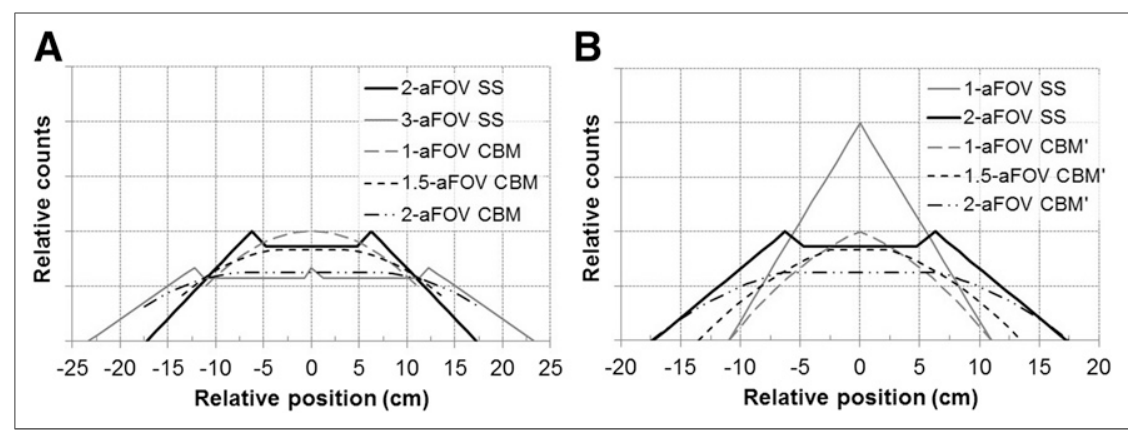

FIGURE 7. Scan length tailor-fitting in SS mode versus $\mathrm{CBM}(\mathrm{A})$ and $\mathrm{CBM}^{\prime}(\mathrm{B})$ modes.

the profile in 1-aFOV CBM (22-cm axial extent) mode; but 2-aFOV SS mode provides longer axial coverage. Similarly, 3aFOV SS mode is preferable to 2-aFOV SS mode. In practice, the superiority of slice sensitivity profiles in SS mode is more apparent compared with the profiles in $\mathrm{CBM}^{\prime}$ mode (Fig. 7). For the same axial coverage and scan duration, SS mode offers higher sensitivity than $\mathrm{CBM}^{\prime}$ mode. Tailor-fitting scan length using $\mathrm{CBM}^{\prime}$ results in lower sensitivity and shorter axial coverage.

The axial coverage in SS mode can, in principle, be finely adjusted by adjusting the amount of bed-position overlap, which trades off axial coverage with sensitivity magnitude and profile (Supplemental Fig. 3). The user's ability to adjust the amount of bed overlap depends on the PET scanner make/model. In the mCT Flow scanner, the bed overlap is $43 \%$ by default and cannot be adjusted. The amount of bed overlap can be optimized on the basis of the desired axial coverage and the imaging task, for example, by minimizing the errors in volumetric quantification (12) across the axial coverage. Decreasing the overlap excessively from the manufacturer's recommendations could decrease the sensitivity and increase the image noise in overlap regions, especially for large patients.

In this study, the comparison of SS mode and CBM $\left(\mathrm{CBM}^{\prime}\right)$ modes was made by fixing the acquisition scan duration. For a given acquisition duration, SS mode offers higher overall sensitivity than $\mathrm{CBM}$ and $\mathrm{CBM}^{\prime}$ modes. The findings in this study are still valid for shorter acquisition durations, that is, the relative sensitivity profiles do not change for a 3-min scan.

Our findings are also applicable for scanners with different length of aFOV. In this study, our scanner has an aFOV of $22 \mathrm{~cm}$. For scanners with different aFOVs, the relative relationship of SS and CBM modes is expected to be similar, although the gradients of the sensitivity profiles will change.

In this work, we presented simplified models to characterize the counting efficiencies across the aFOV in both SS mode and CBM mode. Even though these idealized models do not incorporate details (2) about data acquisition (axial compression, maximum acceptance angle, and number of segments used), correction, and image reconstruction, they were still effective for demonstrating the difference in counting efficiency between SS mode and CBM mode.

Importantly, the lack of attenuation information outside the CBMmode aFOV may be overcome by more advanced reconstruction algorithms (e.g., data-driven algorithms) or simply by altering the CBM-mode scan protocol (e.g., matching the CT scan prescription to the PET prescription with overscan regions). The use of an attenuation-corrected PET signal from outside the CBM-mode scan prescription would help improve the $\mathrm{CBM}^{\prime}$ slice sensitivity profile to CBM slice sensitivity profile, which resembles that in 2aFOV SS mode in the central region (as shown in Fig. 3A). Nevertheless, we showed that 2-aFOV SS is still favorable because of its longer axial coverage while having higher overall slice sensitivity.

The primary objective of this study was to optimize the acquisition protocol for posttherapy microsphere ${ }^{90} \mathrm{Y}$ PET/CT studies; however, we used a ${ }^{68} \mathrm{Ge}$ phantom as a surrogate radionuclide. ${ }^{68} \mathrm{Ge}$ and ${ }^{90} \mathrm{Y}$ PET studies may show differences in absolute slice sensitivity, scatter properties, singles, random coincidence, and resolution; nevertheless, the relative counting efficiency in different acquisition modes should be similar between different radionuclides. Also, using ${ }^{68} \mathrm{Ge}$ instead of ${ }^{90} \mathrm{Y}$ allowed us to acquire higher counts in a shorter duration with lower radioactivity.

\section{CONCLUSION}

The ability of CBM mode to tailor-fit the PET/CT scan length and local scan duration is not realized in studies with a short scan length $(\leq 30 \mathrm{~cm})$ and a long scan duration ( $\geq 20 \mathrm{~min} / \mathrm{aFOV}$ for the Biograph mCT Flow scanner). The SS acquisition mode is preferable to CBM mode for limited-view organ and count-starved scans, such as ${ }^{90} \mathrm{Y}$ PET/CT liver scans, because of the higher slice sensitivity of SS mode, which leads to better image quality and quantification precision.

\section{DISCLOSURE}

Research reported in this publication was supported in part by the National Cancer Institute of the National Institutes of Health under award number R01CA138986. The University of Texas MD Anderson Cancer Center is supported in part by the National Cancer Institute through Cancer Center support grant P30CA016672. No other potential conflict of interest relevant to this article was reported.

\section{ACKNOWLEDGMENTS}

We thank Drs. Maurizio Conti and Vladimir Y. Panin from Siemens Healthcare (Knoxville, TN) for their assistance with 
retrieving the slice sensitivity profiles from step-andshoot and continuous-bed-motion data acquisitions. We also thank the Department of Scientific Publication, UT MD Anderson Cancer Center for assistance with manuscript editing.

\section{REFERENCES}

1. Dahlbom M, Yu D-C, Cherry SR, Chatziioannou A, Hoffman EJ. Methods for improving image quality in whole body PET scanning. IEEE Trans Nucl Sci. 1992;39:1079-1083.

2. Panin VY, Smith AM, Hu J, Kehren F, Casey ME. Continuous bed motion on clinical scanner: design, data correction, and reconstruction. Phys Med Biol. 2014;59:6153-6174.

3. Schubert SF, Pajevic S, Carson RE. Whole body PET using overlapped 3D acquisition and weighted image summation. IEEE Nucl Sci Symp Conf Rec. 1996;2:1285-1289.

4. Dahlbom M, Reed J, Young J. Implementation of true continuous 2D/3D whole body PET scanning. IEEE Nucl Sci Symp Conf Rec. 2000;3:17/13$17 / 17$.
5. Everding M, Emery D, Mawlawi O, et al. Impact of continuous bed motion (CBM) PET/CT scanners on clinical operation. J Nucl Med. 2014;55(suppl 1):2511.

6. Schatka I, Weiberg D, Reichelt S, et al. A randomized, double-blind, crossover comparison of novel continuous bed motion versus traditional bed position whole-body PET/CT imaging. Eur J Nucl Med Mol Imaging. 2016;43:711-717.

7. Osborne DR, Acuff S, Cruise S, et al. Quantitative and qualitative comparison of continuous bed motion and traditional step and shoot PET/CT. Am J Nucl Med Mol Imaging. 2014;5:56-64.

8. Carlier T, Eugène T, Bodet-Milin C, et al. Assessment of acquisition protocols for routine imaging of Y-90 using PET/CT. EJNMMI Res. 2013;3:11.

9. Elschot M, Lam MGEH, van den Bosch MAAJ, Viergever MA, de Jong HWAM. Quantitative Monte Carlo-based ${ }^{90} \mathrm{Y}$ SPECT reconstruction. J Nucl Med. 2013;54:1557-1563.

10. Daube-Witherspoon ME, Muehllehner G. Treatment of axial data in threedimensional PET. J Nucl Med. 1987;28:1717-1724.

11. Bailey DL. Data acquisition and performance characterization in PET. In: Bailey DL, Townsend DW, Valk PE, Maisey MN, eds. Positron Emission Tomography. London, U.K.: Springer-Verlag; 2005.

12. Siman W, Mawlawi OR, Mikell JK, Mourtada F, Kappadath SC. Effects of image noise, respiratory motion, and motion compensation on 3D activity quantification in count-limited PET images. Phys Med Biol. 2017;62: 448 . 